\title{
Optimization and performance validation for the fully automatic blood type analysis system
}

\author{
Rongrong Pang, Libo Zhang, Yong Wang, Wenping Han, Min Huang, Qiang Fu* \\ Nanjing Red Cross Blood Center, Nanjing, Jiangsu 210003, China
}

\begin{abstract}
To evaluate and validate the application of fully automatic blood type analysis system parameters under actual lab conditions. All key system parameters were optimized and validated accordingly. The optimized parameters were centrifugal speed at $550 \mathrm{rpm}$; centrifugal time $20 \mathrm{~min}$; resuspension speed 1,200 rpm; resuspension time: $45 \mathrm{~s}$; incubation temperature $25^{\circ} \mathrm{C}$; incubation time $400 \mathrm{~s}$; incubation rate: $0 \mathrm{rpm}$. The sampled red blood cell concentration was $3 \%$. The ratio of plasma to red blood cells reagent was $60 \mu \mathrm{L}: 30 \mu \mathrm{L}$; the ratio of antibody (reagent) to sampled diluted red blood cell was $30 \mu \mathrm{L}: 30 \mu \mathrm{L}$. After applying our key parameters for optimization and validation, the automatic blood type detection system's performance was found to meet the relevant requirements, effectively improving the accuracy and reliability of the detection system.
\end{abstract}

Keywords: blood type, performance validation, parameters

\section{INTRODUCTION}

In recent years, both the traditional manual method and semi-automatic methods of adding samples for blood group screening and identification have been phased out in blood-bank blood group detection, in favor of the automatic blood group detection sys$t \mathrm{em}^{[1,2]}$. The optimization of these new systems is a prerequisite for their accurate operation in immunohematology laboratories. However, most automatic blood group analyzers are of the open detection type, which miss the relevant parameters for blood group detection reagents while only including the relevant hardware test parameters recommended by equipment manufacturers. At present, despite commercial blood group detection reagents being suitable for various detection methods, with reagent instructions providing reagent-to-blood cell or plasma reaction ratios, definite volumes suitable for automatic blood group systems are still lacking ${ }^{[3]}$.

Orthogonal design is a popular experimental method that provides a convenient, fast and costsaving resolution for optimization in many fields. We successfully applied orthogonal design to determine the optimal parameters combination for the Microlab STAR BG analytical system, which has potential to be a guide for future optimizing operations, with possible application to optimization related issues with other automated blood typing analyzers.

\section{MATERIALS AND METHODS}

\section{Sample source}

Vacuum blood sampling tubes containing anticoagulants were used for collecting of $4 \sim 5 \mathrm{~mL}$ venous blood from each volunteer donor at our center, which

*Correspondence to: Qiang Fu, Nanjing Red Cross Blood Center, Nanjing, Jiangsu 210003, China. TEL: 0086-25-83476311; E-mail: fuqiangnj@ hotmail.com.

Conflict of interests: The authors declared no conflict of interests. 
were centrifuged for $15 \mathrm{~min}$ at $1,500 \mathrm{~g}$. Three tubes of $\mathrm{A}_{2} \mathrm{~B}$ blood type samples were obtained from Beijing Jinhao Pharmaceutical Co., Ltd., China.

\section{Reagents}

Anti-A and anti-B blood grouping reagents (lot number 20180801021) were provided by Shanghai Blood Biomedical Co., Ltd., China. Anti-D blood grouping reagent (lot number BMK1509D) was provided by Millipore Co., UK. Human blood type A, B, O cells reagents (lot number 20180801021) used for reverse typing, were provided by Beijing Jinhao Pharmaceutical Co., Ltd., China.

\section{Instruments}

The automatic blood group system (STAR-BG) was provided by Hamilton Co., Swiss.

\section{Study flowchart}

The study flowchart was seen in Fig.1.

\section{Parameter identification}

Test parameters were divided into fixed param-

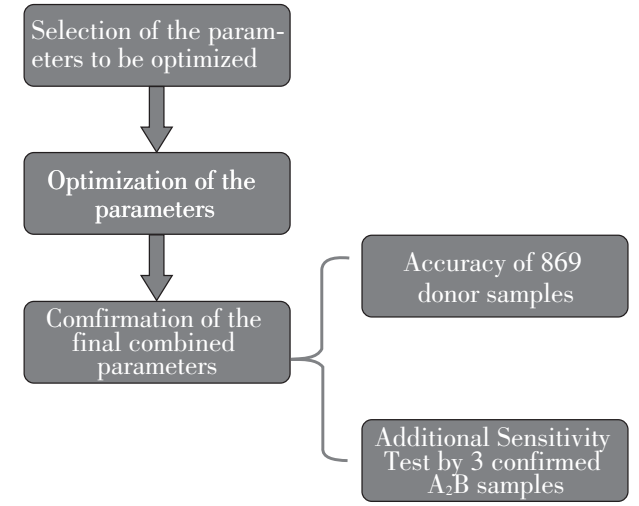

Fig.1 The study flowchart

eters and optimal parameters. Mixing conditions, centrifugal conditions and incubation temperature were set according to fixed parameters recommended by the manufacturers. Incubation rate, diluted erythrocyte sample loading volume, and monoclonal antibodies for forward typing, and the ratio of plasma to erythrocyte reagent in reverse typing were selected as parameters to be optimized, as shown in Table 1.

Table 1 Parameters to be optimized

\begin{tabular}{|c|c|c|c|c|}
\hline Parameter name & $\begin{array}{l}\text { Equipment set- } \\
\text { table range }\end{array}$ & $\begin{array}{l}\text { Equipment manufac- } \\
\text { turer recommended } \\
\text { parameters }\end{array}$ & $\begin{array}{l}\text { Reagent manufacturer recommended } \\
\text { parameters }\end{array}$ & Final determined parameters \\
\hline Mixing time(s) & $80-160$ & 120 & 1 & 120 \\
\hline Centrifugal speed(rpm) & $100-2,500$ & 550 & 1 & 550 \\
\hline Centrifugal time & $0-30 \mathrm{~min}$ & $20 \mathrm{~min}$ & $\begin{array}{l}\text { Instructions 2.6: The reaction can be en- } \\
\text { hanced when placed at room temperature } \\
{\left[(23 \pm 3){ }^{\circ} \mathrm{C}\right] \text { for a few minutes, but the time }} \\
\text { should not exceed } 30 \text { min (forward typing } \\
\text { reagent); incubation at room temperature for } \\
15-20 \text { min(anti-D). }\end{array}$ & $20 \mathrm{~min}$ \\
\hline Incubation temperature( $\quad$ ) & $15-30$ & 25 & 1 & 25 \\
\hline Scattered time(s) & $30-60$ & 45 & 1 & 45 \\
\hline Incubation speed(rpm) & $0-500$ & $0-500$ & 1 & to be optimized \\
\hline Incubation time(s) & $0-500$ & 400 & 1 & 400 \\
\hline $\begin{array}{l}\text { Erythrocyte sample con- } \\
\text { centration }\end{array}$ & 1 & 1 & 1 & $3 \%$ \\
\hline $\begin{array}{l}\text { Erythrocyte reagent loading } \\
\text { volume (reverse typing) }\end{array}$ & 1 & 1 & $\begin{array}{l}\text { Instructions recommend the ratio of plasma } \\
\text { to cells reagent be } 1: 1\end{array}$ & $\begin{array}{l}\text { The ratio of plasma to eryth- } \\
\text { rocyte needs to be optimized }\end{array}$ \\
\hline $\begin{array}{l}\text { Plasma sample loading vol- } \\
\text { ume (reverse typing) }\end{array}$ & 1 & 1 & $\begin{array}{l}\text { Instructions recommend the ratio of plasma } \\
\text { to cells reagent be } 1: 1\end{array}$ & $\begin{array}{l}\text { The ratio of plasma to eryth- } \\
\text { rocyte needs to be optimized }\end{array}$ \\
\hline $\begin{array}{l}\text { Anti-A, anti-B, anti-D } \\
\text { reagent loading volume } \\
\text { (forward typing and } \mathrm{RH} \text { ) }\end{array}$ & 1 & 1 & $\begin{array}{l}\text { Instructions 2.1: add a drop of anti-A or } \\
\text { anti-B reagent; } \\
\text { Instruction 4.2: add a drop of anti-D reagent }\end{array}$ & $\begin{array}{l}\text { The ratio of plasma to eryth- } \\
\text { rocyte can be } 1: 1 \text {, but sam- } \\
\text { pling quantity needs to be } \\
\text { optimized. }\end{array}$ \\
\hline
\end{tabular}




\section{Parameter optimization}

For the three factors mentioned in Table 1 to be optimized, the mixed-level orthogonal design was carried out. Based on the recommended range of 0-500 rpm given by the instrument manufacturer, the parameters of incubation speed were set at five levels: $0 \mathrm{rpm}$, $150 \mathrm{rpm}, 300 \mathrm{rpm}, 450 \mathrm{rpm}$ and $500 \mathrm{rpm}$. The sample size of diluted red blood cells and monoclonal antibodies in forward typing was set at three levels: $30 \mu \mathrm{L}: 30 \mu \mathrm{L}$, $40 \mu \mathrm{L}: 40 \mu \mathrm{L}$, and $50 \mu \mathrm{L}: 50 \mu \mathrm{L}$, based on the recommendation of reagent manufacturer $1: 1$. The ratio of plasma to erythrocyte reagent was set at $30 \mu \mathrm{L}: 30 \mu \mathrm{L}$, $60 \mu \mathrm{L}: 30 \mu \mathrm{L}$, and $90 \mu \mathrm{L}: 30 \mu \mathrm{L}$. According to different parameters, 25-day experiments were carried out. Grouping accuracy was served to evaluate the performance of the optimized condition.

\section{Further optimization of lipemic samples}

According to our optimized parameters, the ratio of plasma to cell reagent in 16 lipemic samples was further fine-adjusted to reduce the influence of lipid blood on blood group detection.

\section{Validation of optimized parameters}

After setting the optimized parameters, the test results were validated, and a retrospective analysis was carried out three months after setting the parameters.

\section{Performance of the detection system after optimization accuracy test}

Totally 869 specimens (283 type A, 246 type B, 256 type $\mathrm{O}, 84$ type $\mathrm{AB}$ ) were tested for forward and reverse typing for 5 consecutive days, and the accuracy of grouping was evaluated. The acceptable accuracy was more than $95 \%$.

\section{Additional sensitivity test according to the Chinese Pharmacopoeia}

Referring to the Chinese Pharmacopoeia's requirement for sensitivity to anti-A reagent, three A2B samples were detected in parallel with routine samples, and the experimental results were recorded to validate the analyzer's sensitivity to difficult blood groups.

\section{Statistical analysis}

SPSS 23.0 software was applied for the orthogonal modeling and data analysis, including direct-viewing analysis and variance analysis. $\mathrm{P}$-values less than 0.05 were regarded as statistically significant.

\section{RESULTS Parametric optimization results}

A total of 25 independent runs (128 samples in each run) of blood groups were carried out in 25 days (data shown in Table 2). The variance analysis showed that the $P$-values of incubation speed and loading volumes of diluted red blood cells and monoclonal antibodies in forward typing were 0.364 and 0.153 , respectively, indicating that these two factors did not significantly influence the ABO grouping, as shown in Table 3, Table 4 and Table 5. However, the $P$-value of the ratio of plasma to erythrocyte reagent was less than 0.001 , indicating that the ratio of plasma to erythrocyte reagent was the major influencing factor, as shown in Table 6. Finally, considering the results of direct viewing analysis and variance analysis, as shown in Table 5, we chose $0 \mathrm{rpm}$ as the optimal incubation speed. For the ratio of plasma to cell reagents, multiple comparisons showed no significant difference between proportion of $90 \mu \mathrm{L}: 30 \mu \mathrm{L}$ and $60 \mu \mathrm{L}: 30 \mu \mathrm{L}$, and the parameters were then optimized in lipemic samples below. To minimize the cost of antibody reagents, $30 \mu \mathrm{L}: 30 \mu \mathrm{L}$ was selected for the forward typing.

\section{Further optimization results for lipemic samples}

The ratio of plasma to cell reagents was tested at 90:30 and 60:30 in 16 lipemic samples. The results showed that two lipemic samples could not be correctly interpreted at 90:30, and the accuracy rate was $87.5 \%$, while the accuracy was $100 \%$ at $60: 30$. There-

Table 2 Schedule of an orthogonal design

\begin{tabular}{|c|c|c|c|c|}
\hline $\begin{array}{c}\text { Test date } \\
\text { (month/day) }\end{array}$ & $\begin{array}{c}\text { Suspension } \\
\text { (reaction) } \\
\text { speed }(\mathrm{rpm})\end{array}$ & $\begin{array}{c}\text { Erythrocyte } \\
\text { sample } \\
\text { volume } \\
(\mu \mathrm{L})\end{array}$ & $\begin{array}{c}\text { Ratio of } \\
\text { plasma to } \\
\text { erythrocyte } \\
(\mu \mathrm{L})\end{array}$ & $\begin{array}{c}\text { Rate of types } \\
\text { A/B/O correctly } \\
\text { interpretation } \\
(\%)\end{array}$ \\
\hline $6 / 14$ & 450 & $50: 50$ & $60: 30$ & 100.0 \\
\hline $6 / 15$ & 300 & $40: 40$ & $30: 30$ & 100.0 \\
\hline $6 / 16$ & 300 & $30: 30$ & $60: 30$ & 100.0 \\
\hline $6 / 19$ & 500 & $40: 40$ & $90: 30$ & 91.7 \\
\hline $6 / 20$ & 150 & $40: 40$ & $60: 30$ & 100.0 \\
\hline $6 / 21$ & 0 & $40: 40$ & $60: 30$ & 100.0 \\
\hline $6 / 26$ & 500 & $30: 30$ & $30: 30$ & 100.0 \\
\hline $6 / 29$ & 150 & $50: 50$ & $30: 30$ & 100.0 \\
\hline $7 / 03$ & 450 & $40: 40$ & $90: 30$ & 82.4 \\
\hline $7 / 04$ & 450 & $40: 40$ & $30: 30$ & 96.9 \\
\hline $7 / 05$ & 150 & $30: 30$ & $60: 30$ & 100.0 \\
\hline $7 / 06$ & 300 & $50: 50$ & $90: 30$ & 55.8 \\
\hline $7 / 07$ & 450 & $40: 40$ & $60: 30$ & 100.0 \\
\hline $7 / 10$ & 0 & $30: 30$ & $90: 30$ & 60.5 \\
\hline $7 / 12$ & 500 & $50: 50$ & $60: 30$ & 99.2 \\
\hline 7/13 & 300 & $30: 30$ & $60: 30$ & 99.2 \\
\hline $7 / 14$ & 0 & $50: 50$ & $30: 30$ & 100.0 \\
\hline $7 / 17$ & 300 & $40: 40$ & $30: 30$ & 100.0 \\
\hline $7 / 18$ & 500 & $30: 30$ & $30: 30$ & 100.0 \\
\hline $7 / 19$ & 500 & $40: 40$ & $60: 30$ & 100.0 \\
\hline $7 / 20$ & 450 & $30: 30$ & $30: 30$ & 100.0 \\
\hline $7 / 21$ & 150 & $40: 40$ & $30: 30$ & 98.9 \\
\hline $7 / 24$ & 0 & $30: 30$ & $30: 30$ & 100.0 \\
\hline $7 / 25$ & 150 & $30: 30$ & $90: 30$ & 56.9 \\
\hline $7 / 26$ & 0 & $40: 40$ & $60: 30$ & 100.0 \\
\hline
\end{tabular}


Table 3 Tests of between-subjects effects

\begin{tabular}{|c|c|c|c|c|c|}
\hline Source & Type III sum of squares & Degree of freedom & Mean square & F value & $P$ value \\
\hline Modified model & $4,052.003^{\mathrm{a}}$ & 8 & 506.500 & 11.489 & $<0.001$ \\
\hline Interception & $162,688.642$ & 1 & $162,688.642$ & $3,690.356$ & $<0.001$ \\
\hline Suspension (reaction) Velocity & 205.148 & 4 & 51.287 & 1.163 & 0.364 \\
\hline Loading volume of erythrocyte sample & 186.267 & 2 & 93.133 & 2.113 & 0.153 \\
\hline Ratio of plasma to erythrocytes reagent & $3,660.588$ & 2 & $1,830.294$ & 41.518 & $<0.001$ \\
\hline Error & 705.357 & 16 & 44.085 & & \\
\hline Total & $224,062.250$ & 25 & & & \\
\hline Revised total & $4,757.360$ & 24 & & & \\
\hline
\end{tabular}

Dependent variable: types $\mathrm{A} / \mathrm{B} / \mathrm{O}$ correctly interpretation rate.

a: $\mathrm{R}^{2}=0.852$ (adjusted $\mathrm{R}^{2}=0.778$ ).

Table 4 Multiple comparisons among different ratios of antibodies and diluted cells

\begin{tabular}{|c|c|c|c|c|c|}
\hline $\begin{array}{l}\text { (I)Volume of antibodies and } \\
\text { cell samples }(\mu \mathrm{L})\end{array}$ & $\begin{array}{c}\text { (J)Volume of antibodies } \\
\text { and cell samples }(\mu \mathrm{L})\end{array}$ & $\begin{array}{c}\text { Difference of mean } \\
\text { value(I-J) }\end{array}$ & Standard error & $P$ value & $95 \%$ confidence interval \\
\hline \multirow[t]{2}{*}{$30: 30$} & $40: 40$ & -5.330 & 2.9693 & 0.092 & $-11.625 \sim 0.965$ \\
\hline & $50: 50$ & 0.660 & 3.6367 & 0.858 & $-7.049 \sim 8.369$ \\
\hline \multirow[t]{2}{*}{$40: 40$} & $30: 30$ & 5.330 & 2.9693 & 0.092 & $-0.965 \sim 11.625$ \\
\hline & $50: 50$ & 5.990 & 3.6367 & 0.119 & $-1.719 \sim 13.699$ \\
\hline $50: 50$ & $30: 30$ & -0.660 & 3.6367 & 0.858 & $-8.369 \sim 7.049$ \\
\hline
\end{tabular}

Dependent variable: types $\mathrm{A} / \mathrm{B} / \mathrm{O}$ correctly interpretation rate.

Based on the actually measured average value.

The error term is mean square (error) $=44.085$.

Table 5 Multiple comparisons among different suspension speed

\begin{tabular}{cccccc}
\hline (I) Suspension (reaction) speed & (J)Suspension (reaction) speed & Difference of mean value(I-J) & Standard error & $P$ value & $95 \%$ confidence interval \\
\hline $0 \mathrm{rpm}$ & $150 \mathrm{rpm}$ & 0.940 & 4.1993 & 0.826 & $-7.962 \sim 9.842$ \\
& $300 \mathrm{rpm}$ & 1.100 & 4.1993 & 0.797 & $-7.802 \sim 10.002$ \\
& $450 \mathrm{rpm}$ & -3.760 & 4.1993 & 0.384 & $-12.662 \sim 5.142$ \\
& $550 \mathrm{rpm}$ & -6.080 & 4.1993 & 0.167 & $-14.982 \sim 2.8822$ \\
$150 \mathrm{rpm}$ & $0 \mathrm{rpm}$ & -0.940 & 4.1993 & 0.826 & $-9.842 \sim 7.962$ \\
& $300 \mathrm{rpm}$ & 0.160 & 4.1993 & 0.970 & $-8.742 \sim 9.062$ \\
& $450 \mathrm{rpm}$ & -4.700 & 4.1993 & 0.280 & $-13.602 \sim 4.202$ \\
\hline & $550 \mathrm{rpm}$ & -7.020 & 4.1993 & 0.114 & $-15.922 \sim 1.882$ \\
& $0 \mathrm{rpm}$ & -1.100 & 4.1993 & 0.797 & $-10.002 \sim 7.802$ \\
& $150 \mathrm{rpm}$ & -0.160 & 4.1993 & 0.970 & $-9.062 \sim 8.742$ \\
& $450 \mathrm{rpm}$ & -4.860 & 4.1993 & 0.264 & $-13.762 \sim 4.042$ \\
\hline & $550 \mathrm{rpm}$ & -7.180 & 4.1993 & 0.107 & $-16.082 \sim 1.722$ \\
& $0 \mathrm{rpm}$ & 3.760 & 4.1993 & 0.384 & $-5.142 \sim 12.662$ \\
\hline & $150 \mathrm{rpm}$ & 4.700 & 4.1993 & 0.280 & $-4.202 \sim 13.602$ \\
& $300 \mathrm{rpm}$ & 4.860 & 4.1993 & 0.264 & $-4.042 \sim 13.762$ \\
\hline $50 \mathrm{rpm}$ & $550 \mathrm{rpm}$ & -2.320 & 4.1993 & 0.588 & $-11.222 \sim 6.582$ \\
& $0 \mathrm{rpm}$ & 6.080 & 4.1993 & 0.167 & $-2.822 \sim 14.982$ \\
\hline & $150 \mathrm{rpm}$ & 7.020 & 4.1993 & 0.114 & $-1.882 \sim 15.922$ \\
\hline & $300 \mathrm{rpm}$ & 7.180 & 4.1993 & 0.107 & $-1.722 \sim 16.082$ \\
\hline & $450 \mathrm{rpm}$ & 2.320 & 4.1993 & 0.588 & $-6.582 \sim 11.222$ \\
\hline
\end{tabular}

Dependent variable: types $\mathrm{A} / \mathrm{B} / \mathrm{O}$ correctly interpretation rate.

Based on the actually measured average value.

The error term is mean square (error) $=44.085$.

fore the ratio of plasma to red blood cell reagents was finally determined as 60:30.

\section{Final optimization of parameters}

Taking both results from direct-viewing analysis and variance analysis into consideration, $0 \mathrm{rpm}$ was selected as the optimal incubation speed and the proportion 60:30 was selected as the optimal ratio for plasma and reagent RBCs. To minimize the cost of antibody reagents, the ratio $30 \mu \mathrm{L}: 30 \mu \mathrm{L}$ was selected for forward typing. The final optimization parameters are shown in Table 7.

\section{Validation results of optimization parameters}

Under optimal conditions obtained from orthogonal design, a set of 322 random donor samples were carried out to confirm the optimized combination, and no erroneous results were found. 
Table 6 Multiple comparisons among different ratios of plasma and RBC reagents

\begin{tabular}{|c|c|c|c|c|c|}
\hline $\begin{array}{l}\text { (I)Volume of plasma and } \\
\text { erythrocyte reagent }(\mu \mathrm{L})\end{array}$ & $\begin{array}{l}(\mathrm{J}) \text { Volume of plasma and } \\
\text { erythrocyte reagent }(\mu \mathrm{L})\end{array}$ & $\begin{array}{c}\text { Difference of mean } \\
\text { value(I-J) }\end{array}$ & Standard error & $P$ value & $95 \%$ confidence interval \\
\hline \multirow[t]{2}{*}{$90: 30$} & $60: 30$ & -0.260 & 2.9693 & 0.931 & $-6.555 \sim 6.035$ \\
\hline & $30: 30$ & $30.120^{*}$ & 3.6367 & $<0.001$ & $22.411 \sim 37.829$ \\
\hline \multirow[t]{2}{*}{$60: 30$} & $90: 30$ & -0.260 & 2.9693 & 0.931 & $-6.035 \sim 6.555$ \\
\hline & $30: 30$ & $30.380^{*}$ & 3.6367 & $<0.001$ & $22.671 \sim 38.089$ \\
\hline \multirow[t]{2}{*}{$30: 30$} & $90: 30$ & $-30.120^{*}$ & 3.6367 & $<0.001$ & $-37.829 \sim-22.411$ \\
\hline & $60: 30$ & $-30.380^{*}$ & 3.6367 & $<0.001$ & $-38.089 \sim-22.671$ \\
\hline
\end{tabular}

Dependent variable: types $\mathrm{A} / \mathrm{B} / \mathrm{O}$ correctly interpretation rate.

Based on the actually measured average value.

The error term is mean square (error) $=44.085$.

* The significant level of difference between mean values is 0.05 .

Table 7 Final optimization of parameters

\begin{tabular}{lc}
\hline \multicolumn{1}{c}{ Name of parameters } & Final determined parameters \\
\hline Mixing speed & $1,200 \mathrm{rpm}$ \\
Mixing time & $120 \mathrm{~s}$ \\
Centrifugal speed & $550 \mathrm{rpm}$ \\
Centrifugal time & $20 \mathrm{~min}$ \\
Incubation temperature & 25 \\
Scattered speed & $1,200 \mathrm{rpm}$ \\
Scattered time & $45 \mathrm{~s}$ \\
Incubation speed & $0 \mathrm{rpm}$ \\
Incubation time & $400 \mathrm{~s}$ \\
Erythrocyte sample concentration & $3 \%$ \\
Loading volume and ratio of plasma and & $60 \mu \mathrm{L}: 30 \mu \mathrm{L}$ \\
erythrocyte reagent (reverse typing ) & \\
Loading volume of antibody reagent and & $30 \mu \mathrm{L}: 30 \mu \mathrm{L}$ \\
diluted erythrocyte sample & \\
\hline
\end{tabular}

\section{Accuracy of test results}

Totally 869 samples (283 type A, 246 type B, 256 type $\mathrm{O}, 84$ type $\mathrm{AB}$ ) were tested with forward and reverse typing for 5 consecutive days. The accuracy of ABO interpretation was more than $99.60 \%$, and the accuracy of $\mathrm{Rh}(\mathrm{D})$ grouping was $100 \%$ (Table 8).

Table 8 Retrospective evaluation results

\begin{tabular}{cccc}
\hline Blood group & Total cases & Correctly grouped cases & Accuracy $(\%)$ \\
\hline Type A & 283 & 283 & 100.0 \\
Type B & 246 & 246 & 100.0 \\
Type O & 256 & 255 & 99.6 \\
Type AB & 84 & 84 & 100.0 \\
Rh(D) & 869 & 869 & 100.0 \\
\hline
\end{tabular}

\section{Additional sensitivity test results according to the Chinese Pharmacopoeia}

Test results of three known $\mathrm{A}_{2} \mathrm{~B}$ samples were all correctly classified as AB type, which met the detection requirements of Chinese Pharmacopoeia for $\mathrm{A}_{2} \mathrm{~B}$ type $^{[4]}$.

\section{Parameter reassessment}

Up to Dec. 8, 2017, we did not receive any adverse feedback related to blood grouping, and no errors were found due to severe lipemic or hemolytic samples.

\section{DISCUSSION}

At present, the automatic blood group analysis system is an open system, which generally has no specified reagents. Although each equipment manufacturer provides the appropriate parameters for their equipment, these parameters need to be optimized when using different reagents. Also the sample status, centrifugal force, manufacturer of reagents, and sample loading volumes are different in each laboratory. Therefore, the setting of system parameters and reagent loading volume must be validated scientifically and systematically, instead of using empirical or estimated values ${ }^{[5,6]}$. Only by validating the parameters affecting the test results can the reliability of the results be ensured, especially for the correct detection of subtypes, weak antigens, weak antibodies and other difficult blood groups, so as to improve the safety of blood transfusion ${ }^{[7,8]}$.

In the validation of the blood group detection system in this paper, the key parameters of the system firstly needed to be analyzed and identified. With regard to the mixing speed, mixing time, the centrifugal speed, and centrifugal time, they were classified as non-key parameters, as the reaction time, speed or time changes had little impact on the results. After scattering and suspending in the reaction process, strong agglutination changed little, but weak agglutination dispersed. The weak agglutination was then identified by the detection system after incubation. The incubation speed, the sample loading volume of diluted red blood cells and monoclonal antibodies in forward typing and the ratio of plasma to red blood cells reagent do have an impact on the blood group results, and were judged as the key parameters of the system. The orthogonal key parameters were analyzed by SPSS software to determine the appropriate parameters and the ratio of antigen to antibody. At the same time, lipemic samples were validated according to the appropriate parameters. So, by means of reducing plasma loading volume and increasing imaging 
transmittance, the interference of lipemic samples on imaging can be avoided.

However, there were still some deficiencies in our work; for example, we did not compare two (or more) sets of blood-typing systems in our laboratory. Also, additional subtype blood samples, such as B subtype should be included to confirm that the system can meet the expected requirements after optimization.

In summary, by bearing in mind the manufacturer's test parameter recommendations, assessing laboratory detection system related samples and reagents simultaneously, and selecting the key parameters for validation and further optimizing the parameters on the basis of validation, the processing ability and reliability of the detection system can be effectively improved. It also provides a method for other laboratories to evaluate the test parameters of their automatic blood group detection system and reagents.

\section{References}

[1] Xu TT, Zhang H, Yu Q, et al. Discussion on the application of PK7300 automatic blood group analyzer in blood stations. Journal of Clinical Hematology (Blood Transfusion and Testing) (in Chinese), 2018, 31(1): 145-7.

[2] Xiao XP. Application of fully automatic digital blood group analyzer in blood group screening. China Medical
Device Information (in Chinese), 2018, 24 (1): 143-4.

[3] Yang ZS, Zhou HY, Feng QX, et al. Establishment and evaluation of non-matching PK7300 automatic blood group detection system. Chinese Journal of Blood Transfusion (in Chinese), 2017, 30 (11): 1228-31.

[4] National Pharmacopoeia Commission. Pharmacopoeia of the People's Republic of China(Chinese edition). Beijing: China Medical Science and Technology Press, 2011.

[5] Wang X, Pan T, Liu J, et al. Performance of PK7300 automatic blood group analysis system was confirmed before use. Chinese Journal of Blood Transfusion (in Chinese), 2017, 30 (9): 1016-8.

[6] Zhang HX, Chen ZH, Wang YB, et al. Confirmation of Galileo Neo automatic blood group analysis system. Journal of Clinical Hematology (Blood Transfusion and Testing) (in Chinese), 2017, 30 (4): 645-6.

[7] Recommendations for evaluation, validation and implementation of new techniques for blood grouping, antibody screening and cross-matching. British Committee for Standards in Haematology, Blood Transfusion Task Force [J]. Transfus Med, 1995, 5 (2): 145-50.

[8] Zhou ZZ, Xu D, Sun L, et al. The progress in pre-transfusion test technique research. Asia-Pacific Journal of Blood Types and Genes, 2017,1(3):7-12.

(Received 08 May 2019, Revised 06 June 2019, Accepted 14 June 2019) 